\section{CELLULAR IMMORTALITY}

\section{Early old age}

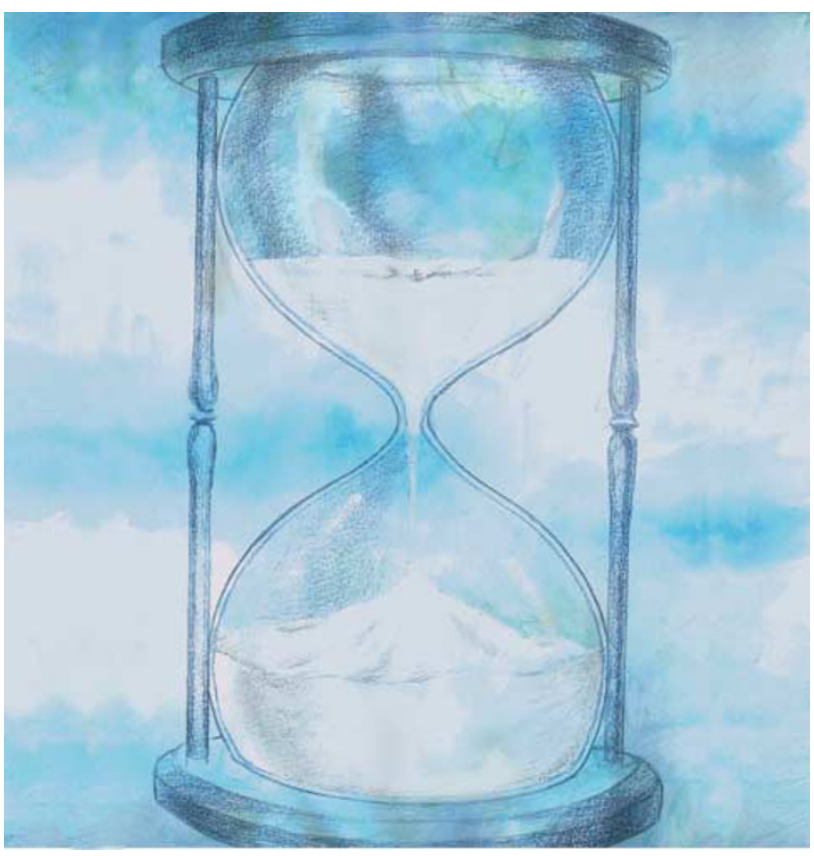

Werner syndrome is an autosomal recessive disease caused by inactivation of the gene encoding the DNA helicase WRN and is characterized by premature ageing, genomic instability and increased non-epithelial cancer incidence. It is thought that the erosion of telomeres - structures that cap chromosomes and are essential for chromosomal stability — has a role in the pathogenesis of this syndrome. Ron DePinho, Sandy Chang and colleagues have developed a mouse model that is null for both Wrn and the RNA component of telomerase $(\operatorname{Terc})$ - an enzyme essential for telomere maintenance - that shows many of the classic features of Werner syndrome.

WRN is involved in DNA recombination, replication and repair, and hyper-recombination and numerous chromosomal aberrations have been observed in individuals with Werner syndrome. DePinho and Chang hypothesized that a combination of impaired DNA repair and telomere dysfunction might drive Werner syndrome pathogenesis.
The authors carried out successive intercrosses between $\mathrm{Wrn}^{-/-}$ mice and $\mathrm{Terc}^{-1-}$ mice to produce cohorts with progressively shorter telomeres and increasing telomere dysfunction. In first- and secondgeneration $\mathrm{Terc}^{-1-}$ mice, Wrn status had no impact on clinical appearance, but the fourth- to sixth-generation $\mathrm{Terc}^{-/-} \mathrm{Wrn}^{-/-}$mice had lower body weights and shorter survival times than $\mathrm{Terc}^{-1-} \mathrm{Wrn}^{+/+}$mice. Although healthy in early life, by $12-16$ weeks of age many of the $\mathrm{Terc}^{-1-} \mathrm{Wrn}^{-1-}$ mice had features of premature ageing, including Werner-syndrome-related diseases. Increased apoptosis in gastrointestinal crypt cells and increased numbers of fused chromosomes in bone-marrow cells were seen in successive generations of $\mathrm{Terc}^{-1-} \mathrm{Wrn}^{-/-}$ mice. This reinforced a link between genomic instability due to WRN loss and telomere dysfunction.

So, how did these genotypes affect the cancer phenotype of these mice? The prematurely aged lategeneration $\mathrm{Terc}^{-1-} \mathrm{Wrn}^{-/-}$mice were

\title{
Receptors and effectors
}

Effective therapies for non-Hodgkin's B-cell lymphoma aim to deplete the B-cell population in patients. However, the precise mechanism by which the humanized immunoglobulin G1 (IgG1) antibody therapy rituximab kills B cells was previously unknown. Jungi Uchida et al. now reveal the mechanism involved.

Rituximab, which targets a B-cell-specific antigen called CD20, could affect many aspects of the immune response, including antibody, effector-cell-(macrophage and natural killer cell) and complementdependent cytoxicity; the disruption of CD20 signalling pathways; and the induction of apoptosis. Previous studies have looked at the mechanisms in vitro or in circulating human B cells only. So, the authors developed a mouse model for antiCD20 immunotherapy using 12 mouse anti-mouse CD20 monoclonal antibodies (mAbs) to study each of the possible mechanisms. All these antibodies bound to $\mathrm{B}$ cells in the CD20 wild-type mice and depleted both the circulating and splenic B-cell compartments. The effectiveness of $\mathrm{mAb}$-induced B-cell depletion correlated closely with $\mathrm{mAb}$ isotype - a single injection of an IgG2a mAb (MB20-11) depleted more than $95 \%$ of blood B cells and more than $93 \%$ of splenic $B$ cells. None of the antibodies had any effect in $\mathrm{Cd} 2 \mathrm{O}^{-/-}$mice.

Immune effector cells express three different Fc receptor classes for IgG. Fc $\gamma$ RI is the highest-affinity receptor and binding of IgG to it triggers phagocytosis by macrophages and cytotoxicity by natural killer cells. Although treatment of mice deficient in either Fc $\gamma$ RI or Fc $\gamma$ RIII with MB20-11 did deplete B cells, treatment of mice deficient in both Fc $\gamma$ RI and Fc $\gamma$ RIII did not deplete $B$ cells. This shows that binding to one of these receptors is important for efficacy of anti-CD20 mAbs. Next, the authors looked at complement-deficient mice to assess the role of complement in B-cell depletion by anti-CD20 mAbs.
In vitro, the antibodies caused B-cell lysis and apoptosis only in the presence of complement. However, in vivo, there was no difference in the ability of any $\mathrm{mAb}$ to induce B-cell killing in the wild-type or complement-knockout mice.

So, Fc receptors are crucial for the efficiency of anti-CD20 mAbs; but what are the effectors of this response? When mice lacking $\mathrm{T}$ cells or natural killer cells were treated with MB20-11, more than $96 \%$ of B cells were depleted. However, similar treatment of macrophage-deficient mice did not cause significant depletion of circulating or splenic B cells.

The authors conclude that a likely mechanism of B-cell depletion by anti$\mathrm{CD} 20 \mathrm{mAbs}$ is Fc $\gamma \mathrm{R}$-mediated phagocytosis of $\mathrm{mAb}$-coated B cells by macrophages. This knowledge should help to understand the response and resistance to rituximab therapy and the development of effective methods to enhance the benefits of therapies for non-Hodgkin's lymphoma. Ezzie Hutchinson

(2) References and links ORIGINAL RESEARCH PAPER Uchida, J. et al. The innate mononuclear phagocyte network depletes $B$ lymphocytes through $\mathrm{Fc}$ receptor-dependent mechanisms during antiCD20 antibody immunotherapy. J. Exp. Med. 199, 1659-1669 (2004) 